\title{
Modeling pavement damage and predicting fatigue cracking of flexible pavements based on a combination of deterministic method with stochastic approach using Miner's hypothesis
}

\author{
Gamaleddine Elnashar $^{1} \cdot$ Rama B. Bhat $^{1} \cdot$ Ramin Sedaghati $^{1}$
}

() Springer Nature Switzerland AG 2019

\begin{abstract}
Distress in flexible pavements has been a problem in many countries due to the increase in road traffic and vehicle loads. One of the most important distress modes in the design of pavements is fatigue cracking. Despite the fact that there have been considerable efforts in recent years in fatigue performance evaluation and the design process of flexible pavements, there is still a need for further studies to overcome the difficulty in predicting fatigue cracking in terms of damage distribution considering the uncertainty associated with the input parameters of pavement life and traffic repetitions. The purpose of this paper is to develop a methodology for modeling pavement damage and predicting fatigue cracking of flexible pavements based on a combination of deterministic method with stochastic approach using Palmgren-Miner's hypothesis. Four predictive models are introduced and used in a comprehensive case study to estimate the fatigue life of the pavement surface layer. The solutions are obtained through numerical integration based on Gaussian quadrature method. The results reveal that pavement damage has a broad range of distribution in which the actual traffic load repetitions has a Poisson distribution, while the traffic repetitions to failure follows a lognormal distribution.
\end{abstract}

Keywords Damage · Fatigue cracking · Flexible pavement · Deterministic · Stochastic · Miner $\cdot$ Poisson process

\section{List of symbols}

A Average annual commercial vehicle per day

C Laboratory to field adjustment factor

$D \quad$ Damage index of fatigue cracking

E Stiffness modulus of the asphalt layer

$F_{\mathrm{j}}(\mathrm{J}) \quad$ Cumulative distribution function of $\mathrm{J}$

$F_{L} \quad$ Conversion factor

$f_{\mathrm{j}}(J)$ Probability density function of $\mathrm{J}$

$h \quad$ Pavement thickness

$K_{T} \quad$ Thickness correction factor

$k_{i} \quad$ Laboratory material coefficients

$L_{f} \quad$ Lateral distribution factor

$r_{t} \quad$ Annual traffic growth rate

$T$ Total number of periods

$V_{a} \quad$ Air void content

$V_{b} \quad$ Volumetric asphalt content

$X \quad$ Actual traffic load repetitions
$Y \quad$ Number of load repetitions to cause failure

$y_{t} \quad$ Design period

$\beta_{i} \quad$ Field calibration coefficients

$\varepsilon_{t} \quad$ Tensile strain at the bottom of the asphalt layer

$\lambda_{x} \quad$ Expected number of occurrences

$\mu_{j} \quad$ Mean value of $j$

$\sigma_{j} \quad$ Standard deviation of $j$

$\sigma_{j}^{2} \quad$ Variance of $j$

\section{Introduction}

Pavement distress, also known as pavement failure, is defined as any signs of break or fracture in the pavement layer, or any indication of poor or undesirable pavement performance [1]. Guo and Prozzi [2] investigated fatigue life of flexible pavements due to repeated

$\checkmark$ Gamaleddine Elnashar, G_elnash@encs.concordia.ca| 'Department of Mechanical, Industrial and Aerospace Engineering (MIAE), Concordia University, Montreal, QC H3G 1M8, Canada. 
loading. There are many types of pavement distress such as fatigue cracking, rutting, potholes, patches, raveling, bleeding, etc. [3]. The previous research revealed that the fatigue cracking, caused by repeated and excessive traffic loading, is the most common distress observed in flexible pavements $[4,5]$.

Fatigue cracking first appears as a set of micro-cracks in the wheel paths, and progresses into a network of interconnecting cracks, and eventually leading to potholes [3]. Fatigue cracking, in flexible pavements, consists of two phases: crack initiation and crack propagation which is caused by tensile strains generated in the pavement due to traffic loading and temperature variations [6]. Crack initiation can be measured using different experiments. Despite the various efforts in testing and measuring the crack initiation and propagation, these attempts still suffer in view of their stochastic nature [6]. Further, there are different severity levels to further define the fatigue cracking. According to the pavement distress survey manual [3], low severity fatigue cracking consists of some connecting cracks, and the cracks are not spalled or sealed with no signs of pumping. Moderate severity is reached when the cracks become interconnected, and the cracks may be slightly spalled and may be sealed, and pumping is not evident. A high severity fatigue cracking occurs when pieces may move when subjected to traffic, cracks may be sealed, and pumping is evident. Fatigue cracking can be classified into two main groups: alligator or bottom up fatigue cracking, longitudinal or top down fatigue cracking, and both may look identical on the pavement surface [6]. Alligator fatigue cracking initiates at the bottom of asphalt layer due to mechanical failure caused by the highest tensile stress and strain then propagates randomly upwards to the surface of pavement [7]. Thin pavement layers are most likely to exhibit bottom up fatigue cracking problems, which makes it a problem often aggravated by cold weather [8]. Longitudinal cracking, conceptually similar to alligator cracking, develops at the surface where high localized tensile stress and strain resulting from tire-pavement interaction exist and propagates downwards to the bottom of asphalt layer. Thick pavement layers are most likely exposed to top bottom fatigue cracking [9].

In this research, a methodology has been developed for modeling the pavement damage and predicting the fatigue cracking of flexible pavements by combining the deterministic and stochastic techniques (using Palmgren-Miner's hypothesis) into a general approach. The developed methodology overcomes the shortcomings associated with the detminustic and stochastic models when they are applied individually.

\section{Pavement performance models}

Pavement performance or damage prediction models are one of the major challenges facing researchers and design engineers. In the sixties and seventies, several studies of pavement response due to repreated loads were carried out based on many laboratory fatigue tests on asphalt pavement. As a result, the fatigue life of the asphalt pavement was established based on a relationship between material coefficients and stress or strain levels induced by repeatedly applied loading [2]. Extensive research has been conducted to model and predict fatigue cracking in the last few years. Based on the prediction results of performance models, these models may be classified as either deterministic or probabilistic. Deterministic models can be divided into mechanistic, empirical and mechanistic-empirical (M-E) models [10, 11]. Mechanistic models are based on the theories of mechanics, in which stresses and strains of a pavement layer can be obtained using simple assumptions and simplifications, such as isotropic, linear-elastic and homogeneous material, small strain and static loading. While these models are simple and can provide the general response behavior of the pavement, they are not practically effective to describe pavement deterioration due to high nonlinearity in the behavior of pavement materials which are not only anisotropic but also depend on time, temperature and other parameters [12]. Empirical models are based on the results of experiments and statistical techniques [13]. They are employed to overcome the limitations of simplified theoretical models used in the mechanistic approach. The empirical models link the pavement distress with the traffic loadings and pavement deflection and provide the number of load repetitions required to cause pavement failure [13]. One of the major disadvantages of the empirical models is that they are experiment based and developed for particular pavemment area and thus cannot be directly applied for different pavement sections. In other words, they can be used only to a particular section area. M-E models combine the mechanistic and the empirical approaches into one general model to take advantage of the merits of each model and also to overcome some of the shortcomings associated with those models once applied individually [13]. In M-E models, the strains generated at the critical locations due to single wheel load of the vehicle are, first, identified, and then the empirical fatigue model can be used to determine the pavement life. Probabilistic models, which predict distribution of events and occurrences, are represented by transition probability process models and reliability analysis to estimate deterioration with age for different combination of variables [10, 11 and 13]. 
One of the first empirical models was reported in 1929 [12]. In the fifties, pavement performance received some attention from several researchers utilizing empirical models. Since then, several empirical models were developed to link pavement performance data to design input parameters. The most common empirical model used worldwide is American Association of State Highway and Transportation Officials (AASHTO) which has been improved many times over the years for different pavements [12].

According to Schwartz and Carvalho [12], the first $M-E$ model was suggested by Kerkhoven and Dormon in 1953. They used a failure method to minimize pavement deterioration based on the vertical compressive strain on the bottom of the asphalt layers. Then in 1960, Saal and Pell suggested another failure criterion to reduce the fatigue cracking based on the horizontal tensile strain at the bottom of the asphalt layers. The first design concept of pavement appeared in 1965 by Dormon and Metcalf [12]. Fatigue life has been expressed based on the relationship between the number of load repetitions to failure and the tensile strains and material properties obtained from laboratory and experimental design through the following equation $[15,16]$ :

$Y=k_{1}\left(\frac{1}{\varepsilon_{t}}\right)^{k_{2}}$

where $Y$ is the number of load applications to failure, $\varepsilon_{t}$ is the horizontal tensile strain at the critical location and $k_{1}$, $k_{2}$ stand for laboratory material coefficients.

Further studies related the fatigue life with the tesile strain $\left(\varepsilon_{t}\right)$, the material characteristic coefficients $\left(k_{1}, k_{2}, k_{3}\right)$ and the stiffness modulus of the asphalt layer, $E$, as given by $[5,6]$ :

$Y=k_{1}\left(\frac{1}{\varepsilon_{t}}\right)^{k_{2}}\left(\frac{1}{E}\right)^{k_{3}}$

Later, in 1975 Pell and Cooper studied the effects of the air void content, $V_{a}$, and the volumetric asphalt content, $V_{b}$, on the fatigue performance of asphalt mixture [2] and added new terms to the Eq. (2) as:

$Y=k_{1}\left(\frac{1}{\varepsilon_{t}}\right)^{k_{2}}\left(\frac{1}{E}\right)^{k_{3}}\left(\frac{V_{b}}{V_{b}+V_{a}}\right)^{k_{4}}$

where $k_{4}$ is another laboratory material coefficient. It has been reported that minimizing the air voids and maximizing the amount of the asphalt was beneficial to fatigue life [15].

Further developments were also conducted by the Asphalt Institute in 1981 and Shell International
Petroleum in 1982 to incorporate strain-based criteria in their M-E methods. Several studies over the past three decades have advanced $M-E$ techniques. Most of the work, however, was based on the Shell and the Asphalt Institute methods. The Asphalt Institute (Al 1982) incorporated a correction term $C$ to express the number of load applications to failure as follows $[2,5,16,17]$ :

$Y=18.4 C\left[0.00432\left(\frac{1}{\varepsilon_{t}}\right)^{3.291}\left(\frac{1}{E}\right)^{0.854}\right]$

where $C$ represents the laboratory to field adjustment factor (correction factor) and can be defined by:

$C=10^{M}$

$M=4.84\left(\frac{V_{b}}{V_{b}+V_{a}}-0.69\right)$

In the nineties a large laboratory effort was undertaken to predict a pavement damage model by researchers at the University of California, Minnesota department of transportation and also Illinois department of transportation. As a result, different models were developed in which the effects of other factors on fatigue life have been considered. In 1990, the National Cooperative Highway Research Program (NCHRP) 1-26 project report integrated most of the studies proposed by previous research work and augmented some environmental parameters, such as asphalt layer temperature, and used Miner's criterion to determine the damage model and predict the fatigue cracking. Sun et al. [18] in the Federal Highway Administration report of the Texas Flexible Pavement System, proposed a modified equation considering the cracking propagation phase as:

$\log (Y)=-3.13+\frac{h}{380}-3.291 \log \left(\varepsilon_{t}\right)-0.854 \log (E)$

where $h$ is the thickness of the asphalt layer.

In 2007, Schwartz and Carvalho [12] published a design guide for the NCHRP 1-37A project including most of the M-E method to predict pavement distresses due to traffic load incorporating the environmental conditions. Moreover, the NCHRP 1-37A project replaced the equivalent single axle load (ESAL) by distribution of vehicular loads. The M-E PDG model used to predict fatigue cracking using the Asphalt Institute method in 1991 was calibrated using 82 LTPP (Long Term Pavement Performance) section data in 24 states across the USA. First, the pavement damage is determined and then the damage is converted into cracked area. Several revised $M-E$ fatigue models have been suggested by other researchers such as El-Basyouny and Witczack, in 2005 
[14]. The number of repetitions to cause failure for a given load can be expressed as follows [12]:

$Y=k_{t}\left[\beta_{1} k_{1} C\left(\frac{1}{\varepsilon_{t}}\right)^{\beta_{2} k_{2}}\left(\frac{1}{E}\right)^{\beta_{3} k_{3}}\right]$

where $k_{t}$ is the thickness correction factor, and given by:

$$
k_{t}=\frac{1}{0.000398+\frac{0.003602}{1+e^{(11.02-3.49 h)}}}
$$

$\beta_{1}, \beta_{2}, \beta_{3}$ represent the field calibration coefficients.

The damage results from a given load is then computed from the number of repititions using Miner's law as follows:

$D=\sum_{i=1}^{T} \frac{x_{i}}{y_{i}}$

where $D$ is the overall cumulative damage up to $T$ in pavement, $T$ represents the total number of periods, $x_{i}$ is the actual traffic for period $i$ and $y_{i}$ stands for the traffic repetitions to cause failure at period $i$.

To convert damage into cracked area, the following expressions are used:

(a) Alligator fatigue cracking (bottom-up fatigue cracking)

$A F C=\left(\frac{6000}{1+e^{\left[C_{1}+C_{2} \log (100 D)\right]}}\right)\left(\frac{1}{60}\right)$

where $A F C$ is the alligator fatigue cracking, $C_{1}=-2 C_{2}$ and $C_{2}=-2.40874-39.748(1+h)^{-2.856}$

(b) Longitudinal fatigue cracking (top-down fatigue cracking)

$L F C=10.56\left(\frac{1000}{1+e^{[7-3.5 \log (100 D)]}}\right)$

where $L F C$ represents the longitudinal fatigue cracking.

While deterministic models have received appropriate attention in improving methods to determine pavement failure, they may not be accurate enough to predict the pavement distress due to the uncertainty and variability of some pavement parameters. Thus, many probabilistic models have been proposed to cover the stochastic nature of the pavement performance. Golabi et al. [19] in 1982 developed a pavement management system to capture the dynamic and probabilistic aspects of pavement maintenance using the Markov chain method. Madanat et al. [20] in 1995 used a structured econometric approach using a joint discrete-continuous model to predict pavement cracking initiation and progression. Further studies have been suggested by several researchers to investigate the pavement deterioration based on Markov chain processes such as that of Li et al. [21] who in 1996 developed a Markov probabilistic method to determine a pavement deterioration rates in which the transition probability matrices are determined based on a reliability analysis using Monte Carlo simulation technique. An improved Markov chain model based on the probability distributions using time-based models was proposed by Mishalani and Madanat [22] in 2002. In 2010, Retherford and McDonald [23] discussed the advantages and disadvantages of reliability methods used in $M-E$ approach for the pavement design.

Other efforts are made for the pavement performance prediction considering the uncertainties and random factors in the pavement deterioration process using techniques such as neural networks, fuzzy logic and hybrid systems [11]. However, these attempts are still in the research and development stage. Despite the various efforts in improving the pavement performance prediction effectively based on either deterministic or probabilistic methods, these attempts suffer from the restrictions associated with the difficulty of considering accurately the dynamic and stochastic nature of pavement distress modes.

\section{Pavement damage and predicting fatigue cracking}

According to the previous studies, the general fatigue damage equation used to predict fatigue cracking life of flexible pavements can be described as [24]:

$Y=k_{1}\left(\frac{1}{\varepsilon_{t}}\right)^{k_{2}}\left(\frac{1}{E}\right)^{k_{3}}$

where $Y$ represents the total equivalent number of load repetitions to cause failure, $\varepsilon_{t}$ is the maximum tensile strain at the bottom of the asphalt layer $\left(\varepsilon_{t}=3.45 \times 10^{-4}\right)$, $E$ stands for the stiffness modulus of the asphalt layer ( $E$ $=3654.22 \mathrm{MPa}$ ) and $k_{1}, k_{2}$ and $k_{3}$ represent the laboratory material coefficient. The Asphalt Institute model is adopted in this study including a correction factor that expresses the uncertainty in the calculation model, in which $k_{1}=1.135 \times 10^{-3}, k_{2}=3.291$ and $k_{3}=0.854$ [24].

Fatigue is considered as a damage accumulation process in which the material property deteriorates continuously under the application of loads. According to Palmgren-Miner's hypothesis, the damage index can be expressed as:

$D=\frac{X}{Y}$ 
where $D$ is the damage index.Y is the traffic repetitions to cause failure and $X$ represents the equivalent number of actual traffic load repetitions applied over the design period of the road section. Considering a compound traffic growth, $X$ can be predicted as follows [25-27]:

$X=365 A \times\left[\frac{\left(1+r_{t}\right)^{y_{t}}-1}{r_{t}}\right] \times F_{L} \times L_{f}$

where $A$ is the average annual commercial vehicle per day $(A=250 \mathrm{cvpd}) . r_{t}$ is the annual traffic growth rate $\left(r_{t}=0.07\right) . F_{L}$ is the vehicle damage factor $\left(F_{L}=2.33\right) . L_{f}$ is the lateral distribution factor $\left(L_{f}=0.75\right)$ and $y_{t}$ is the design period ( $y_{t}=1$ year). $F_{L}$ is used to convert the different vehicular loads into a common axle load, say to a standard axle load of $80 \mathrm{kN}$, and thus, $X$ becomes in terms of standard axle repetitions

The damage index $D$ is considered as the ratio of two random variables $X$ and $Y$ whose joint probability function is defined as:

$f_{X, Y}(x, y)=P(X=x, Y=y)$

where $x>0, y>0$ and $f_{X, Y}(x, y)$ represents the probability that events $X$ and $Y$ occur at the same time.

Since the actual number of traffic load repetitions $X$ is statistically independent of the traffic repetitions to cause failure $Y$, the joint probability of $X$ and $Y$ can be obtained by:

$f_{X, Y}(x, y)=f_{X}(x) f_{Y}(y)$

where $f_{X}(x)$ and $f_{Y}(y)$ are the probability density functions (PDF) of $X$ and $Y$, respectively.

The cumulative distribution function (CDF) of the fatigue damage $D$ is given by:

$F_{D}(d)=P(X \leq Y d)$

Since $X$ and $Y$ are non-negative random variables, then CDF of $D$ can be described as follows:

$F_{D}(d)=\int_{0}^{\infty} \int_{0}^{y d} f_{X}(x) f_{Y}(y) \mathrm{d} x \mathrm{~d} y$

It is known that the probability density function (PDF) of $D$ can be obtained by differentiating the CDF as:

$f_{D}(d)=\frac{\partial F_{D}(d)}{\partial d}$

$f_{D}(d)=\int_{0}^{\infty}\left(\frac{\partial}{\partial d} \int_{x=0}^{y d} f_{X}(x) \mathrm{d} x\right) f_{Y}(y) \mathrm{d} y$

$f_{D}(d)=\int_{0}^{\infty} y f_{X, Y}(y d, y) \mathrm{d} y$
According to Miner's law, the fatigue cracking takes place when damage $D$ reaches or exceeds unity. In other words, the fatigue cracking takes place as the probability of damage index becomes greater than 1 , that is:

$\mathrm{FC}=\mathrm{P}(D>1)=1-\mathrm{P}(D \leq 1)$

$\mathrm{FC}=\int_{1}^{\infty} f_{D}(d) \mathrm{d} d=1-\int_{0}^{1} f_{D}(d) \mathrm{d} d=1-\operatorname{CDF}(D \leq 1)$

where $F C$ represents fatigue cracking.

In order to evaluate the fatigue cracking as given by Eq. (24), the PDF of $D$ is required which are generally unknown, although the majority of the previous research works $[27,28]$ assumed that the pavement damage is either normally or lognormally distributed. In this study, two pavement damage models ( 1 and 2) based on Poisson distribution to characterize the actual traffic load repetitions have been proposed and compared with previously developed models (3 and 4) [25-28].

\subsection{Damage model 1}

Considering the fact that the repeated traffic loads at a given point on the pavement occur continuously and independently of one another, the Poisson process model is a more realistic description of the vehicle traffic, that is $X \sim \operatorname{Pois}\left(\lambda_{X}\right)$, and the corresponding probability mass function (PMF) of $X$ can be expressed as follows:

$f_{X}(x)=\frac{e^{-\lambda_{x}} \lambda_{X}^{x}}{x !}$

where $\lambda_{X}$ is the expected number of occurrences (mean)

Assuming that the mean is a large value, the Poisson distribution maybe approximated by a normal distribution [29] with mean and variance as independent parameters defined as:

$\lambda_{x}=\mu_{x}=\sigma_{x}^{2}$

where $\mu_{x}$ and $\sigma_{x}^{2}$ are the mean value and the variance of $X$, respectively.

The probability density function (PDF) of $X$, then, becomes:

$f_{X}(x)=\frac{1}{\sqrt{2 \pi \lambda_{x}}} e^{-\frac{1}{2}\left(\frac{x-\lambda_{x}}{\sqrt{\lambda_{x}}}\right)^{2}}$

Taking logarithm on both sides of Eq. (13) yields:

$\ln (Y)=\ln \left(k_{1}\right)-k_{2} \ln \left(\varepsilon_{t}\right)-k_{3} \ln (E)$

which means that $\ln (Y)$ is a normally distributed random variable due to the central limit theorem which states that 
the sampling distribution of the mean of any independent, random variable tends toward a normal distribution [30, 31], that is, $\ln (Y) \sim N\left(\mu_{\ln Y}, \sigma_{\ln Y}^{2}\right)$. Thus $Y$ has a lognormal distribution $Y \sim \ln N\left(\mu_{Y}, \sigma_{Y}^{2}\right)$ with PDF as:

$f_{Y}(y)=\frac{1}{y \sigma_{\ln Y} \sqrt{2 \pi}} e^{-\frac{1}{2}\left(\frac{\ln y-\mu \ln x}{\sigma_{\ln X}}\right)^{2}}$

where the mean and variance of $Y$ are given as:

$E(Y)=\mu_{Y}=e^{\mu_{\ln Y}+\left(\sigma_{\ln Y}^{2} / 2\right)}$

$\operatorname{Var}(Y)=\sigma_{Y}^{2}=\left(e^{\sigma_{\ln Y}^{2}}-1\right) e^{2 \mu_{\ln Y}+\sigma_{\ln Y}^{2}}$

In which lognormal distribution parameters are described as:

$\sigma_{\ln Y}^{2}=\ln \left(1+\frac{\sigma_{Y}^{2}}{\mu_{Y}^{2}}\right)$

$\mu_{\ln Y}=\ln \mu_{Y}-\frac{\sigma_{\ln Y}^{2}}{2}=\ln \mu_{Y}-\frac{1}{2} \ln \left(1+\frac{\sigma_{Y}^{2}}{\mu_{Y}^{2}}\right)$

where $\mu_{\ln Y}$ and $\sigma_{\ln Y}^{2}$ are the mean value and variance of $Y$, respectively.

Thus the resulting PDF of the pavement damage in this case can be written as:

$f_{D}(d)=\int_{0}^{\infty} \frac{1}{2 \pi \sigma_{\ln Y} \sqrt{\lambda_{x}}} e^{-\frac{1}{2}\left[\left(\frac{y d-\lambda_{x}}{\sqrt{\lambda_{x}}}\right)^{2}+\left(\frac{\ln y-\mu_{\ln Y}}{\sigma_{\ln Y}}\right)^{2}\right]} d y$

The cumulative distribution function of the pavement can also be expressed as:

$F_{D}(d)=P(D \leq d)=\int_{0}^{d} f_{D}(d) \mathrm{d} d$

$F_{D}(d)=\int_{0}^{d} \int_{0}^{\infty} \frac{1}{2 \pi \sigma_{\ln \gamma} \sqrt{\lambda_{x}}} e^{-\frac{1}{2}\left[\left(\frac{y d-\lambda_{x}}{\sqrt{\lambda_{x}}}\right)^{2}+\left(\frac{\ln y-\mu_{\ln Y}}{\operatorname{In}_{\ln }}\right)^{2}\right]} d y d d$
The fatigue cracking is given by:

$F C=1-\int_{0}^{1} \int_{0}^{\infty} \frac{1}{2 \pi \sigma_{\ln Y} \sqrt{\lambda_{x}}} e^{-\frac{1}{2}\left[\left(\frac{y d-\lambda_{x}}{\sqrt{\lambda_{x}}}\right)^{2}+\left(\frac{\ln y-\mu_{\ln Y}}{\sigma_{\ln Y}}\right)^{2}\right]} d y d d$

\subsection{Damage model 2}

In this model, $X$ is assumed to be a random variable that has a Poisson distribution $X \sim \operatorname{Pois}\left(\lambda_{X}\right)$, and approximated by a normal distribution due to large mean, that is $X \sim \mathrm{N}\left(\lambda_{X}, \lambda_{X}\right)$. Suppose $Y$ follows a normal distribution, that is, $Y \sim \mathrm{N}\left(\mu_{Y}, \sigma_{Y}^{2}\right)$, then the resulting PDF of the pavement damage becomes:

$f_{D}(d)=\int_{0}^{\infty} y \frac{1}{2 \pi \sigma_{Y} \sqrt{\lambda_{x}}} e^{-\frac{1}{2}\left[\left(\frac{y d-\lambda_{x}}{\sqrt{\lambda_{x}}}\right)^{2}+\left(\frac{y-\mu_{Y}}{\sigma_{Y}}\right)^{2}\right]} d y$

According to Fieller [32] and Hinkley [33] the approximate form for such distribution (ratio of two normally distributed random variables) is given by the following expression:

$f_{D}(d)=\frac{b(d) h(d)}{\sqrt{2 \pi \lambda_{x}} \sigma_{y} a^{3}(d)}\left[\Phi\left(\frac{b(d)}{a(d)}\right)-\Phi\left(-\frac{b(d)}{a(d)}\right)\right]+\frac{e^{-\frac{1}{2} c}}{\pi \sigma_{y} \sqrt{\lambda_{x}} a^{2}(d)}$

where

$a(d)=\sqrt{\frac{1}{\lambda_{x}} d^{2}+\frac{1}{\sigma_{y}^{2}}}$

$b(d)=d+\frac{\mu_{y}}{\sigma_{y}^{2}}$

$c=\lambda_{x}+\frac{\mu_{y}^{2}}{\sigma_{y}^{2}}$

$h(d)=e^{\frac{b^{2}(d)-c a^{2}(d)}{2 a^{2}(d)}}$

The corresponding CDF can then be expressed as: 
Subsequently fatigue cracking can be obtained as:

$F C=\int_{1}^{\infty}\left[\frac{b(d) h(d)}{\sqrt{2 \pi \lambda_{x}} \sigma_{y} a^{3}(d)}\left[\Phi\left(\frac{b(d)}{a(d)}\right)-\Phi\left(-\frac{b(d)}{a(d)}\right)\right]+\frac{e^{-\frac{1}{2} c}}{\pi \sigma_{y} \sqrt{\lambda_{x}} a^{2}(d)}\right] d d$

\subsection{Damage Model 3}

According to previous research [25-28] the damage is assumed to follow a normal distribution, $D \sim N\left(\mu_{D}, \sigma_{D}^{2}\right)$, then its PDF, CDF and subsequnetly FC, respectively, can be expressed as:

$f_{D}(d)=\frac{1}{\sigma_{D} \sqrt{2 \pi}} e^{-\frac{1}{2}\left(\frac{d-\mu_{D}}{\sigma_{D}}\right)^{2}}$

$F_{D}(d)=\int_{-\infty}^{d} \frac{1}{\sigma_{D} \sqrt{2 \pi}} e^{-\frac{1}{2}\left(\frac{d-\mu_{D}}{\sigma_{D}}\right)^{2}} d d$

$F C=\Phi\left(\frac{\mu_{D}-1}{\sigma_{D}}\right)=1-\Phi\left(\frac{1-\mu_{D}}{\sigma_{D}}\right)$

here $\mu_{D}$ and $\sigma_{D}$ are the mean value and standard deviation of $D$, respectively. $\Phi$ is the CDF of the standard normal distribution, given by:

$\Phi(u)=\int_{-\infty}^{u} \frac{1}{\sqrt{2 \pi}} e^{-\frac{1}{2} \tau^{2}} \mathrm{~d} \tau$

An approximate expression for $\mu_{D}$ and $\sigma_{D}^{2}$ using Taylor series expansion can be obtained as [34]:

$\mu_{D}=E(D)=E\left(\frac{X}{Y}\right)=\frac{E(X)}{E(Y)} \approx \frac{\mu_{x}}{\mu_{y}} \approx \frac{\lambda_{x}}{\mu_{y}}$

$\sigma_{D}^{2}=\operatorname{Var}(D) \approx \frac{\lambda_{x}}{\mu_{y}^{2}}+\frac{\lambda_{x}^{2} \operatorname{Var}(y)}{\mu_{y}^{4}}$

\subsection{Damage Model 4}

In this model, the damage is assumed to have a lognormal distribution, In $D \sim N\left(\mu_{\ln D}, \sigma_{\ln D}^{2}\right)$, then the pavement damage PDF, CDF and FC, respectively, can be expressed as [25-28]:

$f_{D}(d)=\frac{1}{d \sigma_{\ln D} \sqrt{2 \pi}} e^{-\frac{1}{2}\left(\frac{\ln D-\mu_{\ln D}}{\sigma_{\ln D}}\right)^{2}}$

$F_{D}(d)=\int_{0}^{d} \frac{1}{d \sigma_{\ln D} \sqrt{2 \pi}} e^{-\frac{1}{2}\left(\frac{\ln D-\mu_{\ln D}}{\sigma_{\ln D}}\right)^{2}} \mathrm{~d} d$
$F C=\Phi\left(\frac{\mu_{\ln D}}{\sigma_{\ln D}}\right)=1-\Phi\left(\frac{-\mu_{\ln D}}{\sigma_{\ln D}}\right)$

Here

$\sigma_{\ln D}^{2}=\ln \left(1+\frac{\sigma_{D}^{2}}{\mu_{D}^{2}}\right)$

$\mu_{\ln D}=\ln \mu_{D}-\frac{\sigma_{\ln D}^{2}}{2}=\ln \mu_{D}-\frac{1}{2} \ln \left(1+\frac{\sigma_{D}^{2}}{\mu_{D}^{2}}\right)$

where $\mu_{\ln D}$ and $\sigma_{\ln D}^{2}$ are the mean value and the variance of $\ln D$, respectively.

\section{Numerical results and discussion}

The integrals in the damage models are evaluated numerically using the Gaussian quadrature method implemented using a computer program code developed in the Matlab environment.

Figure 1 shows the PDFs of the pavement damage for different models. It can be seen that the PDF of developed model 2 and model 4 increases significantly with the increase in the damage index in the range between zero and the peak values of $D$, and then decrease sharply with the increase in the damage index, while the PDF of

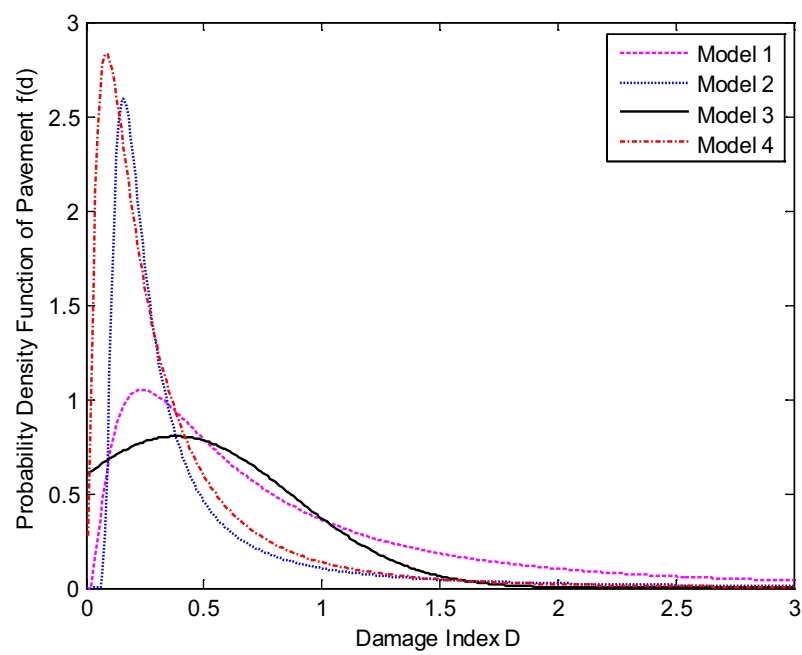

Fig. 1 PDFs of pavement damage for different models 


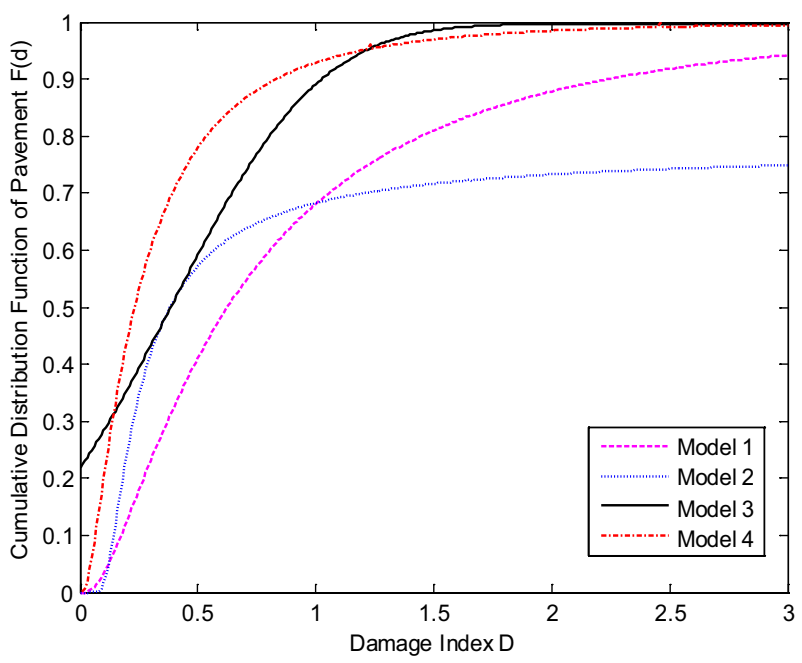

Fig. 2 CDFs of pavement damage for different models

Table 1 Calculated fatigue cracking (FC) for different models

\begin{tabular}{lllll}
\hline & Model 1 & Model 2 & Model 3 & Model 4 \\
\hline FC & 0.3187 & 0.3172 & 0.1035 & 0.0681 \\
\hline
\end{tabular}

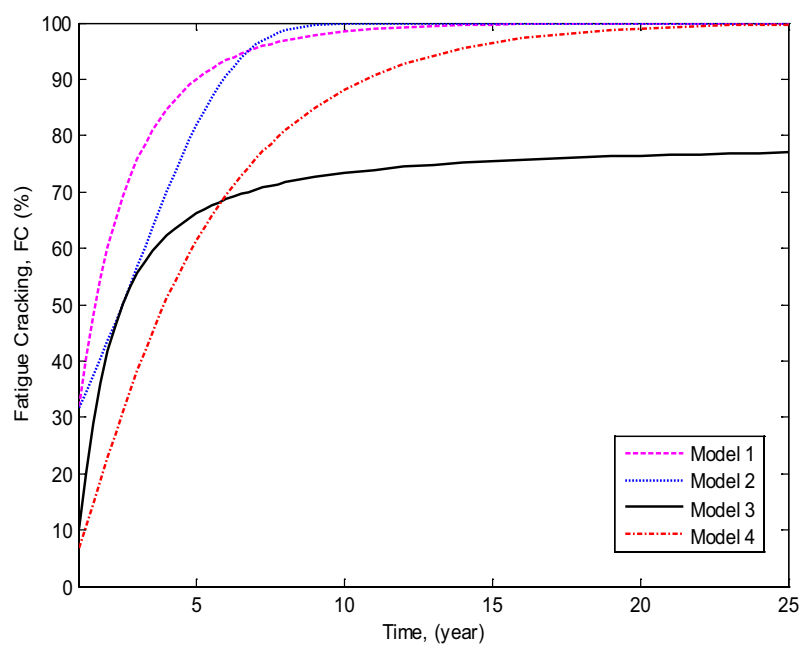

Fig. 3 Fatigue cracking with time for different models

models 1 and 3 tend to decrease gradually, when the damage index increase beyond the peak value. In addition, models 2 and 4 show a relatively narrow range distribution followed by model 3 compared with model 1 that shows a broad range distribution of pavement damage.

Figure 2 shows the cumulative distribution of pavement damage which represents the probability that $D$ will take a value less than or equal to $d(0 \leq d \leq \infty)$, for developed models 1 and 2 compared with models 3 and 4 . It is found that at $D=1$, model 4 shows a highest $\operatorname{CDF}\left(F_{D}(1)=0.9288\right)$, and model 1 gives a lowest CDF $\left(F_{D}(1)=0.6812\right)$, while CDF of models 2 and 3 are $\left(F_{D}(1)=0.6827\right)$ and $\left(F_{D}(1)=0.8913\right)$, respectively. Table 1 provides the fatigue crack index, $\mathrm{FC}=1-\mathrm{CDF}(D=1)$, for different models. As it can be reazlied, developed model 1 shows a higher probability of occurrence of the fatigue cracking followed by models 2 and 3, while model 4 gives a lower expectation of fatigue cracking.

For a long design period (say 25 years) the expected fatigue cracking with time using proposed models is shown in Fig. 3. It can be observed that the expected fatigue cracking using model 1 is higher than the other models in most periods.

The results reveal that the damage distribution is neither normal nor lognormal as the previous research works assumed. According to the derived model (model 1) the damage follows a broad range distribution which represents the distribution of a ratio of two random variables $X$ and $Y$, where $X$ represents the actual traffic load repetitions which follows the Poison distribution, and $Y$ stands for the traffic repetitions to cause fatigue cracking which is lognormally distributed.

\section{Conclusion}

Predicting the fatigue cracking and the pavement damage is presented based on a mechanistic empirical approach using Asphalt Institute model and the probability distribution theory. The Palmgren-Miner's hypothesis is used to estimate the accumulation of damage for flexible pavement based on Poisson distribution to describe the arrival of traffic loads. The solutions are obtained through numerical integration based on Gaussian quadrature method. Major conclusions are:

- The damage distribution is neither normal nor lognormal.

- Damage model 1 has a broad range distribution of pavement damage.

- Model 1 shows the highest expectation of fatigue cracking.

- For a long design period, the expected fatigue cracking using model 1 is higher than those of the other models in most periods.

Thus, the damage distribution is neither normal nor lognormal as the previous research works assumed, and a better estimation of fatigue cracking in asphalt pavements based on damage model can be carried out using model 1 , in which the equivalent actual traffic load repetitions 
has the Poisson distribution, while the traffic repetitions to cause failure follows the lognormal distribution.

Recommendations for future work

- Laboratory and field tests of fatigue cracking should be carried out to validate the accuracy of the proposed damage distribution model.

- Some parameters in this research are considered constant such as the stiffness modulus of the asphalt layer and the related tensile strain. However, they can be stochastic values and follow a known distribution due to variability of certain factors such as environmental conditions.

- Further investigation is needed by considering different traffic distributions.

Acknowledgements Support from Natural Science and Engineering Research Council of Canada (NSERC) is gratefully acknowledged.

\section{Compliance with ethical standards}

Conflict of interest The authors declare that they have no conflict of interest.

\section{References}

1. Erlingsson S (2013) Failure modes in pavements. KTH AF2903 Road Construction and Maintenance AF2903 Road Construction and Maintenance

2. Guo R, Prozzi JA (2009) Prediction of in-service fatigue life of flexible pavements based on accelerated pavement testing. J Test Eval 37(5):449-453

3. Anonymous (2010) Pavement distress survey manual, Oregon Department of Transportation Pavement Services

4. Wang Y, Mahboub KC, Hancher DE (2005) Survival analysis of fatigue cracking for flexible pavements based on long-term pavement performance data. J Transp Eng 131(8):608-616

5. Priest AL, Timm DH (2006) Methodology and calibration of fatigue transfer functions for mechanistic empirical flexible pavement design. No. NCAT Report 06-03

6. Kim YR, Park HJ (2015) Investigation of primary causes of fatigue cracking in asphalt pavement in north Carolina, Research and development, North Carolina State University, Project 2010-01

7. Moreno-Navarro F, Rubio-Gámez MC (2016) A review of fatigue damage in bituminous mixtures: understanding the phenomenon from a new perspective. Constr Build Mater 113:927-938

8. Guercio MC, Mehta Y, McCarthy LM (2015) Evaluation of fatigue cracking performance of asphalt mixtures under heavy static and dynamic aircraft loads. Constr Build Mater 95:813-819

9. Ambassa Z, Allou F, Petit C, Eko RM (2012) Top-down and bottom-up fatigue cracking of bituminous pavements subjected to tangential moving loads. In: 7th RILEM international conference on cracking in pavements 675-685. Springer, Dordrecht

10. Lytton RL (1987) Concepts of pavement performance prediction and modeling. In: Proceedings of 2 nd North American conference on managing pavements, $\mathrm{vol} 2$

11. Ferreira A, Picado-Santos LD, Wu Z, Flintsch G (2011) Selection of pavement performance models for use in the Portuguese PMS. Int J Pavement Eng 12(1):87-97
12. Schwartz CW, Carvalho RL (2007) Evaluation of mechanisticempirical design procedure, Final report, MDSHA Project No. SP0077B41, Maryland (2)

13. Li Z (2005) A probabilistic and adaptive approach to modeling performance of pavement infrastructure. Dissertation, University of Texas

14. El-Basyouny M, Witczak M (2005) Part 2: flexible pavements: calibration of alligator fatigue cracking model for 2002 design guide. Transp Res Rec J Transp Res Board 1919:76-86

15. Read JM (1996) Fatigue cracking of bituminous paving mixtures. Dissertation, University of Nottingham

16. Pokorski P, Radziszewski P, Sarnowski M (2016) Fatigue life of asphalt pavements on bridge decks. Procedia Eng 153:556-562

17. Chua KH, Der Kiureghian A, Monismith CL (1992) Stochastic model for pavement design. J Transp Eng 118(6):769-786

18. Sun L, Hudson WR, Zhang Z (2003) Empirical-mechanistic method based stochastic modeling of fatigue damage to predict flexible pavement cracking for transportation infrastructure management. J Transp Eng 129(2):109-117

19. Golabi K, Kulkarni RB, Way GB (1982) A statewide pavement management system. Interfaces 12(6):5-21

20. Madanat S, Bulusu S, Mahmoud A (1995) Estimation of infrastructure distress initiation and progression models. J Infrastruct Syst 1(3):146-150

21. Li N, Xie WC, Haas R (1996) Reliability-based processing of Markov chains for modeling pavement network deterioration. Transp Res Rec J Transp Res Board 1524:203-213

22. Mishalani RG, Madanat SM (2002) Computation of infrastructure transition probabilities using stochastic duration models. $J$ Infrastruct Syst 8(4):139-148

23. Retherford J, McDonald M (2010) Reliability methods applicable to mechanistic-empirical pavement design method. Transp Res Rec J Transp Res Board 2154:130-137

24. Dilip DM, Ravi P, Babu GS (2013) System reliability analysis of flexible pavements. J Transp Eng 139(10):1001-1009

25. Kalita K, Rajbongshi $P$ (2015) Variability characterisation of input parameters in pavement performance evaluation. Road Mater Pavement Des 16(1):172-185

26. Maji A, Das A (2008) Reliability considerations of bituminous pavement design by mechanistic-empirical approach. Int J Pavement Eng 9(1):19-31

27. Thongram S, Rajbongshi $P$ (2016) Probability and reliability aspects in pavement engineering. National Institute of Technology Silchar

28. Sun L, Hudson WR (2005) Probabilistic approaches for pavement fatigue cracking prediction based on cumulative damage using Miner's law. J Eng Mech 131(5):546-549

29. Griffin TF (1992) Distribution of the ratio of two poisson random variables. Dissertation, Texas Tech University

30. Kallianpur G, Krishnaiah PR, Ghosh JK (1982) A proof of the central limit theorem motivated by the Cramér-Rao inequality. Statistics and probability: essays in honor of CR Rao 141

31. The central limit theorem (2017) http://www.math.uah.edu/stat/ sample/CLT.html. Accessed 01 Jan 2017

32. Fieller EC (1932) The distribution of the index in a normal bivariate population. Biometrika 24:428-440

33. Hinkley DV (1969) On the ratio of two correlated normal random variables. Biometrika 56(3):635-639

34. Van Kempen GM, Van Vliet LJ (2000) Mean and variance of ratio estimators used in fluorescence ratio imaging. Cytomet J Int Soc Anal Cytol 39(4):300-305

Publisher's Note Springer Nature remains neutral with regard to jurisdictional claims in published maps and institutional affiliations. 